\title{
Assessment, Definition, and Classification of Lower Limb Ulcers in Systemic Sclerosis: A Challenge for the Rheumatologist
}

\author{
Jelena Blagojevic, Guya Piemonte, Laura Benelli, Francesca Braschi, Ginevra Fiori, \\ Francesca Bartoli, Serena Guiducci, Silvia Bellando Randone, Felice Galluccio, Laura Cometi, \\ Sergio Castellani, Maria Boddi, Alberto Moggi Pignone, Laura Rasero, Francesco Epifani, \\ and Marco Matucci-Cerinic
}

\begin{abstract}
Objective. To evaluate pathogenesis and clinical features of lower limb ulcers in systemic sclerosis (SSc) and to propose a classification that could be used in clinical practice.

Methods. Charts of 60 patients with SSc who had lower limb cutaneous lesions were reviewed. All patients had videocapillaroscopy and arterial and venous lower limb color Doppler ultrasonography (US). Arteriography was performed if occlusive peripheral arterial disease was suspected.

Results. The 554 lesions were classified as hyperkeratosis, ulcers, and gangrenes. There were 341 $(61.6 \%)$ hyperkeratoses, $208(37.5 \%)$ ulcers, and $5(0.9 \%)$ gangrenes. Ulcers were divided into pure ulcers, ulcers associated with hyperkeratosis, and ulcers secondary to calcinosis. Involvement of arterial and venous macrocirculation as determined by color Doppler US was observed in 17 (18.3\%) and $18(30 \%)$ patients, respectively. Seventeen out of 37 patients with pure ulcers $(45.9 \%)$ presented neither venous insufficiency nor hemodynamically significant macrovascular arterial disease. In these patients, pure ulcers were most likely caused by isolated SSc-related microvascular involvement (pure microvascular ulcers). The only significant risk factor for development of pure microvascular ulcers in the multivariate analysis was the history of lower limb ulcers (OR 26.67, 95\% CI 2.75-259.28; $\mathrm{p}<0.001)$.

Conclusion. Results of our study indicate that lower limb ulcers in SSc often have a multifactorial pathogenesis that may be difficult to manage. Further studies are needed to validate the proposed classification and to assess the most appropriate management of lower limb ulcers in SSc. (First Release February 1 2016; J Rheumatol 2016;43:592-8; doi:10.3899/jrheum.150035)
\end{abstract}

Key Indexing Terms:

SYSTEMIC SCLEROSIS LOWER LIMB ULCERS PATHOGENESIS CLASSIFICATION

Cutaneous ulcers complicate systemic sclerosis (SSc) frequently, are found early in the disease ${ }^{1,2}$, and represent a heavy burden for patients ${ }^{3}$.

In SSc, upper limb ulcers have been evaluated and characterized thoroughly ${ }^{4}$, but there are few studies of lower limb

From the University of Florence: Department of Clinical and Experimental Medicine, Division of Rheumatology; Department of Internal Medicine; Interinstitutional Department of Didactics, Florence, Italy.

J. Blagojevic, MD, Doctoral Candidate, University of Siena and University of Florence Joint Doctoral Program, Department of Clinical and Experimental Medicine, Division of Rheumatology, University of Florence; G. Piemonte, BSN, RN, Doctoral Candidate, University of Florence, Department of Clinical and Experimental Medicine, Division of Rheumatology; L. Benelli, BSN, RN, Registered Nurse, Department of Clinical and Experimental Medicine, Division of Rheumatology, University of Florence; F. Braschi, BSN, RN, Registered Nurse, Department of Clinical and Experimental Medicine, Division of Rheumatology, University of Florence; G. Fiori, MD, Department of Clinical and Experimental Medicine, Division of Rheumatology, University of Florence; F. Bartoli, MD, Department of Clinical and Experimental Medicine, Division of Rheumatology, University of Florence; S. Guiducci, PhD, Researcher, Department of Clinical and Experimental Medicine, Division of Rheumatology, University of Florence; S. Bellando ulcers $5,6,7$. These studies were performed on small series of patients involving limited numbers of lesions. Moreover, the studies neither assessed the lesions' morphologic characteristics, nor proposed a classification. It is important to note that the treatment of lower limb ulcers often represents an

Randone, PhD, Researcher, Department of Clinical and Experimental Medicine, Division of Rheumatology, University of Florence; F. Galluccio, $M D$, Department of Clinical and Experimental Medicine, Division of Rheumatology, University of Florence; L. Cometi, MD, Rheumatology Resident, Department of Clinical and Experimental Medicine, Division of Rheumatology, University of Florence; S. Castellani, PhD, Associate Professor, Department of Clinical and Experimental Medicine, University of Florence; M. Boddi, PhD, Associate Professor, Department of Clinical and Experimental Medicine, University of Florence; A. Moggi Pignone, PhD, Associate Professor, Department of Internal Medicine, University of Florence; L. Rasero, BSN, RN, Associate Professor, Department of Clinical and Experimental Medicine, University of Florence; F. Epifani, $M S$, Coordinator of the Services for Didactics and Educational Management of the University of Florence; M. Matucci-Cerinic, PhD, Professor, Department of Clinical and Experimental Medicine, Division of Rheumatology, University of Florence.

Address correspondence to Dr. J. Blagojevic, Villa Monna Tessa, Viale Pieraccini 18, Florence, Italy.E-mail: jelena308@hotmail.com Accepted for publication November 16, 2015. 
important clinical challenge because of frequent and concurrent involvement of the arterial and venous macrocirculation.

The aim of our study was to evaluate the pathogenesis and clinical features of lower limb ulcers in SSc and to propose a classification that could be used in clinical practice.

\section{MATERIALS AND METHODS}

Charts of 60 patients with SSc seen by the Ulcer Clinic of the Rheumatology Division of University Hospital in Florence (Italy) because of lower limb cutaneous ulcers during a 5-year period (2008-2013) were reviewed, and data were extracted from the standard forms in those charts. All patients fulfilled 2013 American College of Rheumatology/European League Against Rheumatism classification criteria ${ }^{8}$ and were divided into limited (lcSSc) and diffuse (dcSSc) cutaneous subsets ${ }^{9}$. No patient had overlap syndrome with other connective tissue diseases. Our clinic adopts a clinical form to record the natural history of the ulcers, their characteristics, dimensions, recurrence, and type of medications applied. In our center, ulcers are evaluated and classified by 3 nurses specialized in wound healing and 2 rheumatologists with interest in wound healing who are involved in the patients' clinical management. In addition, rheumatologists evaluated ulcers for the presence of overt infection and whether there was a delay in the time to healing or whether complications occurred. Clinical forms were filled out by nurses and checked by rheumatologists.

All patients had videocapillaroscopy and arterial and venous lower limb color Doppler ultrasonography (US) according to the routine protocol adopted by our institution for the management of lower limb lesions in SSc.

In addition, the results of pulmonary function tests, high-resolution computed tomography lung scans, echocardiography, and renal echo Doppler performed on a regular basis within routine management of patients with SSc in our institution were recorded. Relevant clinical data were also collected from patients' history and other case-specific examinations (Appendix 1).

Involvement of arterial macrocirculation was assessed by arterial color Doppler US. Hemodynamically important arterial stenosis was diagnosed if stenosis was between 50-99\%, as described ${ }^{10}$ (Appendix 1) ) $^{10,11,12,13}$. Patients with hemodynamically significant peripheral arterial disease on arterial color Doppler US, with a distribution consistent with the ulcer site, underwent contrast angiography (arteriography), for which a digital subtraction angiography technique was used. The modified Brewster classification was used to classify macrovascular arterial disease detected by angiography ${ }^{14}$ (Appendix 1). Venous insufficiency was evaluated by venous duplex imaging combining B-mode imaging of the deep and superficial veins with pulsed Doppler flow assessment, as described ${ }^{12,13}$ (Appendix 1). Lymphedema was suspected at clinical examination, supported by suggestive US findings when lymphatic lakes and ectasic lymphatic vessels were observed $^{15}$.

At the time of presentation, lower limb lesions were defined as follows: (1) hyperkeratosis - hypertrophy of the stratum corneum of the skin, located in areas subject to increased friction, mainly represented by calluses and corns (Figure 1a); (2) ulcer - loss of tissue involving, in different degrees, the epidermis, dermis, subcutaneous tissue, and sometimes bone; (3) calcinosis - deposits of calcium phosphate in soft tissues, visible to the naked eye and/or confirmed by radiograph; (4) gangrene - defined as necrosis of soft tissues caused by a total lack of blood supply. Dry gangrene presents macroscopically as shrunken and black tissue (Figure 1d). Wet gangrene presents as a moist and infected tissue.

Ulcers were classified into subsets according to their origin and main features: (1) ulcers associated with hyperkeratosis - loss of tissue is usually hidden below the hyperkeratotic tissue and may easily raise suspicion, because it is characterized by inflammation and edema of perilesional skin (Figure 1b); (2) ulcers secondary to calcinosis - loss of tissue caused by a mechanical action of calcinosis erupting through the skin (Figure 1c); and
(3) pure ulcers - loss of soft tissues not occurring in association with hyperkeratosis or in association with calcinosis (Figure 2a-c).

The following criteria, described in the literature ${ }^{16,17,18}$, have been used to define venous, arterial, and microvascular ulcers: (1) venous ulcers, occurring in the gaiter area of the leg, particularly over the medial malleolus, have an irregular, gently sloping edge and are associated with varicosities, edema, venous dermatitis and/or lipodermatosclerosis; (2) arterial ulcers (typically occurring over the toes, heels, and bony prominences of the foot; usually have well-demarcated edges, cold surrounding skin, and often a necrotic base; and are usually associated with intermittent claudication, worsening pain following leg elevation, reduced or abolished peripheral pulses); and (3) microvascular ulcers, defined as ulcers with morphological characteristics of arterial ulcers in the absence of signs of macrovascular arterial impairment (as absent or diminished peripheral pulses).

We did not assess the morphologic and clinical features of either ulcers associated with hyperkeratosis or those secondary to calcinosis, to determine whether they fit criteria for arterial, venous, or microvascular ulcers.

Statistical analysis was done with SPSS software, version 17 (Appendix 1).

\section{RESULTS}

Patients'features and macrovascular involvement. Sixty SSc patients with lower limb lesions attended our ulcer clinic from 2008 to 2013.

About 1000 patients with SSc were followed in our center over the same time period, so we can estimate that about $6 \%$ of our SSc population presented lower limb ulcers.

Clinical features of the study population are summarized in Table 1. Involvement of arterial macrocirculation as determined by color Doppler US was observed in 17 patients $(18.3 \%)$ and of venous macrocirculation as determined by color Doppler US in 18 patients (30\%; Table 1). There were 2 patients with hemodynamically significant peripheral macrovascular arterial disease and concomitant venous insufficiency. Macrovascular involvement was more frequent in the lcSSc subset: arterial disease was present in 16/40 patients (40\%) with lcSSc and $1 / 20$ patients (5\%) with dcSSc; venous involvement was present in $15 / 40$ patients with $1 \mathrm{cSSc}$ $(37.5 \%)$ and $3 / 20$ patients with dcSSc (15\%).

Six patients with hemodynamically significant peripheral arterial disease, identified by color Doppler US in the vascular area above the ulcer site, underwent lower limb arteriography. Of those 6 patients, 2 did not have atherosclerotic risk factors and 4 did. In patients without atherosclerotic risk factors, type IV Brewster disease was observed (diseased distal vessels below the knee with normal vessels above the knee), while all 4 patients with atherosclerotic risk factors had type III Brewster disease (multilevel disease with both proximal and distal arterial lesions with severe involvement of tibial and peroneal arteries).

Lymphedema was observed in 13 patients (21.7\%). Two patients had type II diabetes mellitus, both optimally controlled by oral antidiabetics, and none of them presented diabetic neuropathy. Both presented hemodynamically significant peripheral arterial disease.

Cutaneous lesions. A total of 554 lesions were observed in 60 patients. Eighteen patients (30\%) presented only hyperkeratoses and never developed ulcers, 41 patients $(68.3 \%)$ 

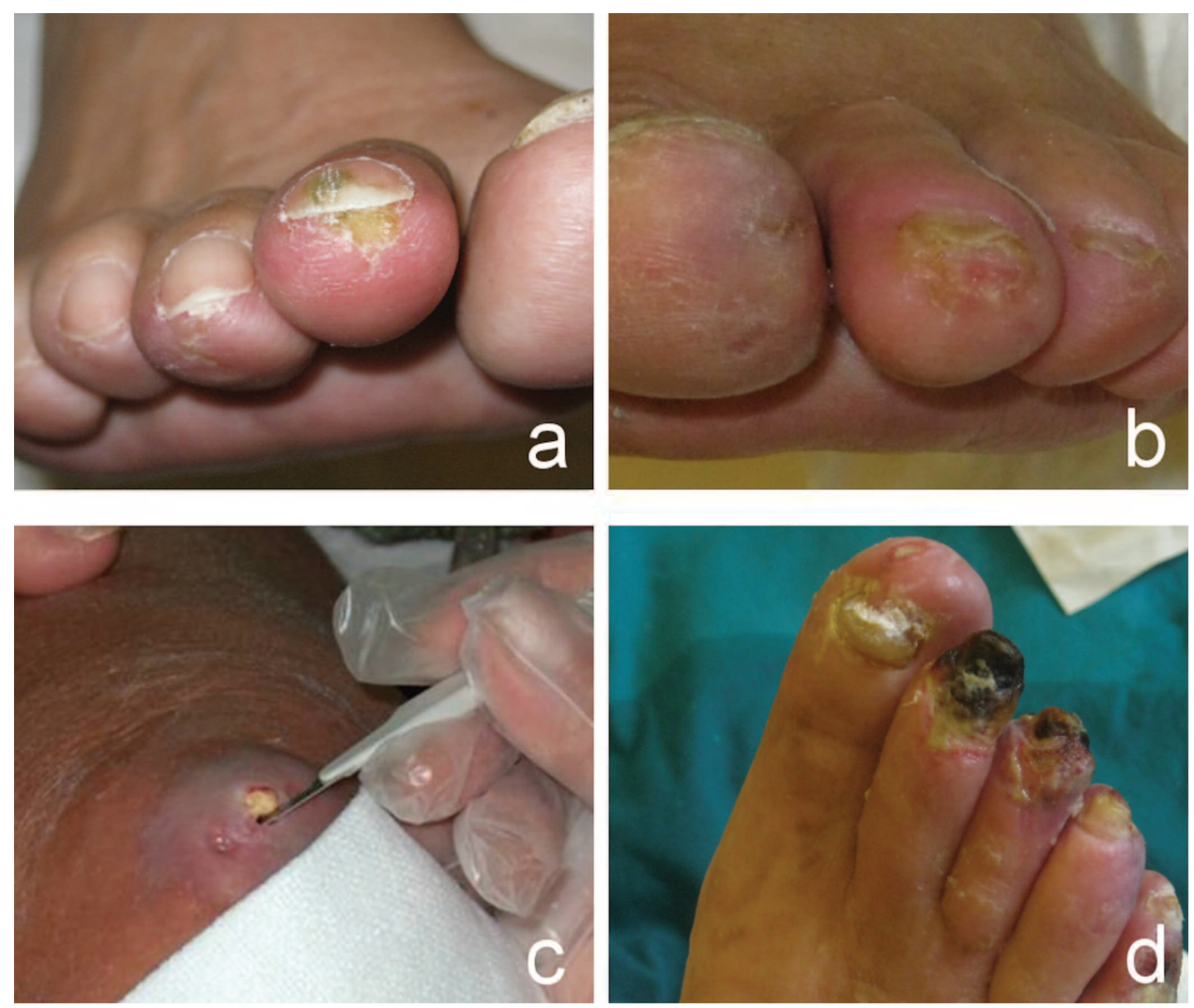

Figure 1. Lower limb lesions. (a) Hyperkeratosis. (b) Ulcer secondary to hyperkeratosis. (c) Ulcer secondary to calcinosis. (d) Gangrene.
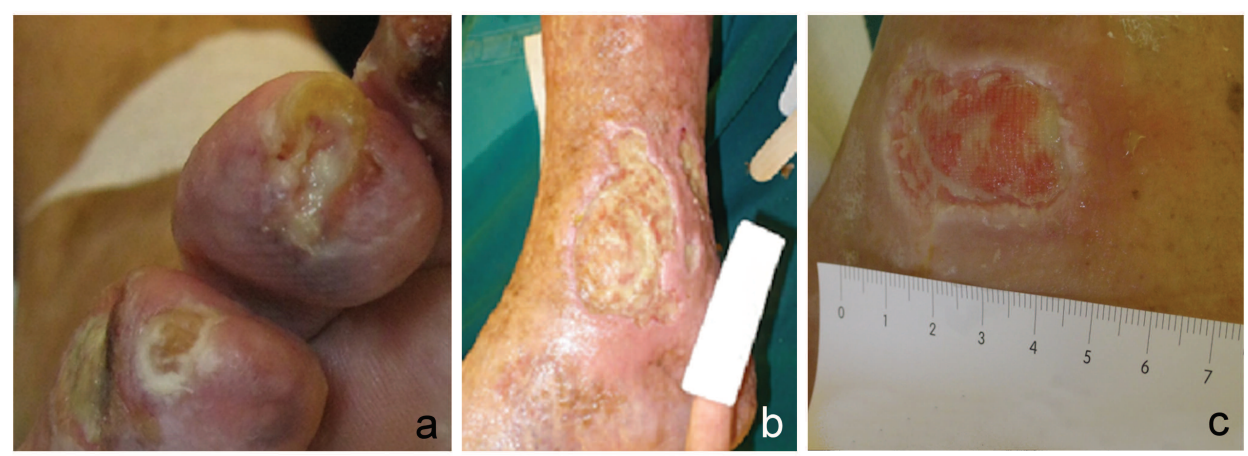

Figure 2. Pure ulcers. (a) Pure ulcer in SSc patient with arterial macrovascular disease. (b) Pure ulcer in SSc patient with macrovascular arterial and venous involvement. (c) Pure ulcer in SSc patient without macrovascular involvement (pure microvascular ulcer). SSc: systemic sclerosis.

had at least 1 ulcer, and $3(5 \%)$ had gangrene. Among patients with ulcers, $37 / 41(90.2 \%)$ had pure ulcers, $6 / 41(14.6 \%)$ had ulcers associated with hyperkeratosis, and 9/41 (21.9\%) had ulcers secondary to calcinosis.

Because all patients with SSc present disease-related microangiopathy, we presumed that ulcers not secondary to hyperkeratoses or to calcinosis might present pure microvascular origin related to $\mathrm{SSc}$; arterial macrovascular origin related to significant arterial macrovascular stenosis, over-lapping microvascular SSc-related etiology; venous macrovascular origin, overlapping SSc-related microangiopathy; or mixed macrovascular arterial-venous origin overlapping SSc-related microangiopathy.

Classification and distribution of lower limb cutaneous lesions are shown in Table 2.

All pure ulcers in patients with hemodynamically signifi-

Personal non-commercial use only. The Journal of Rheumatology Copyright (c) 2016. All rights reserved. 
Table 1. Study population: clinical features and macrovascular involvement. Data are n $(\%)$ unless otherwise indicated.

\begin{tabular}{|c|c|}
\hline Age, yrs, mean \pm SD & $65.5 \pm 14$ \\
\hline \multicolumn{2}{|l|}{ Sex } \\
\hline Female & $53(88.3)$ \\
\hline Male & $7(11.7)$ \\
\hline \multicolumn{2}{|l|}{ Subset } \\
\hline Limited & $40(66.7)$ \\
\hline Diffuse & $20(33.3)$ \\
\hline $\mathrm{RP}$ duration, yrs, mean $\pm \mathrm{SD}$ & $22.6 \pm 13.4$ \\
\hline Disease duration, yrs, mean \pm SD & $15.3 \pm 8.7$ \\
\hline \multicolumn{2}{|l|}{ Autoantibodies } \\
\hline $\mathrm{ACA}$ & $24(40)$ \\
\hline Scl70 & $23(38.3)$ \\
\hline $\mathrm{ACA}+\mathrm{Scl} 70$ & $2(3.3)$ \\
\hline Other & $11(18.4)$ \\
\hline \multicolumn{2}{|l|}{ Videocapillaroscopy } \\
\hline Early & $9(15)$ \\
\hline Active & $18(30)$ \\
\hline Late & $33(55)$ \\
\hline $\mathrm{mRSS}$ & $9.8 \pm 10.2$ \\
\hline \multicolumn{2}{|l|}{ Smokers } \\
\hline Yes & $7(11.7)$ \\
\hline No & $48(80)$ \\
\hline Ex-smoker & $5(8.3)$ \\
\hline Arterial hypertension & $31(51.7)$ \\
\hline \multicolumn{2}{|l|}{ Hypercholesterolemia } \\
\hline Increased total cholesterol & $10(16.7)$ \\
\hline Increased LDL cholesterol & $20(33.3)$ \\
\hline Low HDL cholesterol & $9(15)$ \\
\hline Hypertriglyceridemia & $10(16.7)$ \\
\hline Diabetes mellitus & $2(3.3)$ \\
\hline Intermittent claudication & $10(16.8)$ \\
\hline Upper limb ulcers & $48(80)$ \\
\hline Ulcers in the other sites & $14(23.3)$ \\
\hline Calcinosis & $22(36.7)$ \\
\hline Gastrointestinal involvement & $60(100)$ \\
\hline GERD & $60(100)$ \\
\hline Other & $9(15)$ \\
\hline Interstitial lung disease & $32(53.3)$ \\
\hline Pulmonary arterial hypertension & $9(15)$ \\
\hline Peripheral arterial disease & $17(18.3)$ \\
\hline Not hemodynamically significant & $7(41.2)$ \\
\hline Hemodynamically significant & $10(58.8)$ \\
\hline Venous insufficiency & $18(30)$ \\
\hline
\end{tabular}

ACA: anticentromere autoantibodies; mRSS: modified Rodnan skin score; LDL: low-density lipoprotein; HDL: high-density lipoprotein; GERD: gastroesophageal reflux disease; RP: Raynaud phenomenon.

cant macrovascular arterial disease met morphological criteria for arterial ulcers, described above (Figure 2a).

Only 17 out of 52 lesions (32.7\%) in patients with venous insufficiency and without macrovascular arterial involvement on color Doppler US met the morphological features of venous ulcers described above. Out of 52 pure ulcers (42.3\%), 22 were located on the toes, had morphological features of arterial ulcers, but were not associated with signs of macrovascular arterial impairment (as diminished pulses) and thus were likely related to SSc microangiopathy.

Two patients with concomitant venous insufficiency and hemodynamically significant arterial macrovascular disease presented a total of 16 pure ulcers, but only 1 of them met the criteria for venous ulcers (located in the medial malleolar area, site of venous dermatitis), while others were more likely microvascular/arterial lesions (Figure 2b).

Seventeen out of 37 patients with pure ulcers (45.9\%) presented neither venous insufficiency nor hemodynamically significant macrovascular arterial disease assessed by color Doppler US. Hence, in these patients, pure ulcers were most likely due to SSc-related microvascular involvement only (pure microvascular ulcers).

There was a total of 76 pure ulcers in these patients: 23 (30.3\%) were proximal (above ankle, including ankle) and $53(69.7 \%)$ were distal (below ankle). Proximal lesions were observed more frequently in the lateral malleolar region [12/23 (52.2\%)], pretibial region [4/23 (17.4\%)] and medial malleolar region $[3 / 23(13 \%)]$, while distal lesions were observed on the toes [38/53 (71.7\%)], soles [13/53 (24.5\%)], and feet $[2 / 53(3.8 \%)]$. Pure ulcers in these patients had morphological characteristics of arterial ulcers, but were not associated with absent pulses and/or intermittent claudication (Figure 2c).

When we compared patients with pure microvascular ulcers to patients with pure ulcers related to macrovascular involvement, patients with macrovascular involvement were significantly older $(78.2 \pm 10.6$ vs $60.2 \pm 17.2 \mathrm{yrs}$, respectively, $\mathrm{p}=0.016)$, had more frequently lcSSc $(83.2 \% \mathrm{vs}$ $42.1 \%$, respectively, $\mathrm{p}=0.007$ ), had significantly longer duration of Raynaud phenomenon ( $29.5 \pm 13.4$ vs $18.1 \pm 12.7$ yrs, respectively, $\mathrm{p}=0.018$ ), and had more frequently arterial hypertension (68.4\% vs $27.8 \%$, respectively, $\mathrm{p}=0.044$ ).

When we considered all patients, those with and without ulcers, and excluded patients with pure ulcers of macrovascular origin, risk factors for development of pure ulcers due to isolated microvascular involvement were represented by: dcSSc (OR 4.19, 95\% CI 1.10-15.90; $\mathrm{p}=0.035$ ); history of lower limb ulcers (OR 30.86, 95\% CI 3.34-283.82; p < $0.001)$; presence of ulcers in the other parts of the body excluding finger digital ulcer (DU), such as elbows, forearms, and ears (OR 4.61, 95\% CI 1.01-21.07; $\mathrm{p}=0.049$ ); and systemic steroid treatment at the time of ulcer occurrence (OR 4.80, 95\% CI 1.03-22.29; $\mathrm{p}=0.045)$, in univariate analysis. Upper limb DU alone did not represent a significant risk factor for development of pure microvascular ulcers in the lower limbs. The history of lower limb ulcers remained the only statistically significant risk factor in the multivariate analysis (OR 26.67; 95\% CI 2.75-259.28, p < 0.001).

Age, duration of Raynaud phenomenon and SSc, disease subset, capillaroscopic pattern, and other disease characteristics did not represent risk factors for development of microvascular pure ulcers.

Moreover, there was no difference in the use of vasodilators or immunosuppressants (cyclophosphamide, methotrexate, azathiophrine, and mycophenolate mofetil) between

Personal non-commercial use only. The Journal of Rheumatology Copyright @ 2016 . All rights reserved. 
Table 2. Classification and distribution of lower limb cutaneous lesions in SSc.

\begin{tabular}{lcc}
\hline Type of Lesion & No. Patients Affected and \% of Overall Patients & No. Lesions and \% of Overall Lesions \\
\hline Hyperkeratoses & $23 / 60(38.3)$ & $341 / 554(61.6)$ \\
Ulcers & $41 / 60(68.3)$ & $208 / 554(37.5)$ \\
Pure ulcers secondary to: & $37 / 60(61.7)$ & $162 / 208(77.8)$ \\
Isolated SSc-related microangiopathy & $17 / 37(45.9)$ & $76 / 162(46.9)$ \\
Arterial macrovascular disease + SSc-related microangiopathy & $4 / 37(10.8)$ & $18 / 162(11.1)$ \\
Venous insufficiency + SSc-related microangiopathy & $14 / 37(37.8)$ & $52 / 162(32.1)$ \\
Venous insufficiency + arterial macrovascular disease + SSc-related & & \\
$\quad$ microangiopathy & $2 / 37(5.5)$ & $16 / 162(9.9)$ \\
Ulcers associated with hyperkeratosis & $6 / 60(10)$ & $23 / 208(11.1)$ \\
Ulcers secondary to calcinosis & $9 / 60(15)$ & $23 / 208(11.1)$ \\
Gangrene & $3 / 60(5)$ & $5 / 554(0.9)$ \\
\hline
\end{tabular}

SSc: systemic sclerosis.

patients who had and did not have ulcers and between patients with microvascular and macrovascular ulcers.

We observed dry gangrene of the fingertips in 3 patients. It was secondary to microvascular involvement in 1 patient $(33.3 \%)$ and to significant macrovascular arterial disease in $2(66.7 \%)$.

Table 3 compares features of lower limb cutaneous lesions with features of upper limb DU of corresponding type. Data on the upper limbs have been extrapolated from our previous work on a different study population of patients with $\mathrm{SSc}^{4}$. Because digital pitting scars (DPS) in the upper limbs were defined as small-scale hyperkeratosis, we compared characteristics of ulcers derived from DPS to those of ulcers associated with hyperkeratosis in the lower limbs. Both in the upper and in the lower limbs, pure ulcers were defined as ulcers not occurring in association with DPS or with calcinosis.

\section{DISCUSSION}

Our work clearly demonstrates that lower limb ulcers are a significant burden for patients with SSc. For this reason, we have provided real-life data in an effort to assess their pathogenesis and to propose a classification useful for both clinical practice and clinical trials, as previously done for $\mathrm{DU}^{4}$.

The results of our study show that heterogeneous cutaneous lower limb lesions may be observed in SSc. Estimated prevalence of lower limb lesions in SSc in our study was around $6 \%$ over the observational period, lower than the prevalence of finger DU in $\mathrm{SSc}^{19}$. However, our data demonstrate that although lower limb lesions seem to be less frequent, they are much more difficult to manage than superior limb DU. Comparison with upper limb DU described in our previous study ${ }^{4}$ indicates that lower limb lesions take longer to heal and have higher rates of infection and amputation than superior limb DU of corresponding type. We did not analyze different types of upper limb DU in this study population.

We classified lower limb lesions into 4 types (hyperkeratoses, ulcers, calcinosis, and gangrene). Subsequently, ulcers have been classified as those secondary to hyperkeratosis, secondary to calcinosis, and pure ulcers (i.e., ulcers neither secondary to calcinosis nor associated with hyperkeratosis).

Results of our study show that in SSc patients with lower limb ulcers, concomitant macrovascular arterial and/or venous disease may overlap with SSc-related microangiopathy in a substantial proportion of patients. This has to be taken into account when these lesions are managed.

We observed hemodynamically significant macrovascular arterial involvement, confirmed by arteriography, in $10 \%$ of patients, represented by type III and type IV (Brewster modified classification). In agreement with Dick, et $a l^{14}$, we observed type IV disease in SSc patients without traditional risk factors, suggesting primary macrovascular arterial involvement in SSc, not correlated to atherosclerosis, as described ${ }^{5,20,21}$.

Table 3. Lower limb ulcers versus upper limb digital ulcers.

\begin{tabular}{|c|c|c|c|c|c|c|}
\hline \multirow[t]{2}{*}{ Ulcer Type } & Lower Limbs & Upper Limbs ${ }^{4}$ & Lower Limbs & Upper Limbs ${ }^{4}$ & Lower Limbs & Upper Limbs ${ }^{4}$ \\
\hline & \multicolumn{2}{|c|}{ Time to Healing (weeks) } & \multicolumn{2}{|c|}{ Infection } & \multicolumn{2}{|c|}{ Amputation } \\
\hline $\begin{array}{l}\text { Ulcers associated with hyperker } \\
\text { (lower limbs)/ ulcers derived }\end{array}$ & & & & & & \\
\hline from DPS (upper limbs) & $15.3 \pm 12$ & $3.7 \pm 2.2$ & 0 & 0 & 0 & 0 \\
\hline Pure ulcers, mean \pm SD & $25.6 \pm 23.5$ & $10.9 \pm 9.1$ & $48.1 \%$ & $19.2 \%$ & $1.9 \%$ & $1.7 \%$ \\
\hline $\begin{array}{l}\text { Ulcers secondary to calcinosis, } \\
\text { mean } \pm \text { SD }\end{array}$ & $55.9 \pm 44.5$ & $13.4 \pm 8.5$ & $61.9 \%$ & $40.9 \%$ & 0 & 0 \\
\hline
\end{tabular}

DPS: digital pitting scars. 
In our cohort, venous pathology was detected in $30 \%$ of patients. It is interesting to note that $40 \%$ of ulcers in patients with venous insufficiency had features of microvascular lesions, and thus were most likely due to SSc-related microangiopathy.

Progressive loss of lymphatic vessels in skin of patients with SSc has been described ${ }^{22}$. However, there are no data on prevalence of lymphedema in patients with SSc. We observed lymphedema in $20 \%$ of patients.

In our study, there was a substantial subpopulation of patients who presented pure ulcers but did not have macrovascular involvement (45.9\%); thus we presumed that pure ulcers in these patients were due to SSc-related microangiopathy. These lesions were more frequently distal (below the ankle) and had morphological characteristics of arterial ulcers, but were not associated with absent pulses and/or intermittent claudication.

Pure microvascular ulcers were more frequent in dcSSc than in lcSSc, as reported for DU of the upper limbs in $\mathrm{SSc}^{19}$. However, when we considered all pure ulcers together (both microvascular and macrovascular), pure microvascular ulcers were more frequent in $1 \mathrm{cSSc}$ than in dcSSc $(63.2 \%$ and $36.8 \%$ of patients, respectively). This is because macrovascular involvement is more frequent in $\mathrm{lcSSc}$ that in $\mathrm{dcSSc}$, as suggested by our results.

Risk factors for development of pure ulcers from isolated microvascular involvement in univariate analysis were: $\mathrm{dcSSc}$, history of pure ulcers in the lower limbs, presence of ulcers in the other parts of the body excluding finger DU, and systemic steroid treatment at the time of ulcer occurrence. A history of lower limb ulcers remained the only significant risk factor in multivariate analysis. Interestingly, the upper limb DU alone did not represent risk factors for development of pure microvascular ulcers in the lower limbs.

Some ulcers observed in our study did not seem to have pure vascular pathogenesis because they were related to hyperkeratosis or were consequences of calcinosis. This is in accordance with data observed in our previous study on upper limb ulcers ${ }^{4}$.

The main limitations of the study are the retrospective design and the fact that not all the patients had arteriography, but only those with hemodynamically significant peripheral arterial disease on arterial color Doppler US.

Another important limitation is that we did not evaluate interobserver reproducibility of our classification, although in our clinical practice it seems to be very high.

The results of our study indicate that lower limb ulcers in SSc often have a multifactorial pathogenesis that should be taken into account when dealing with these lesions. Moreover, they may be more difficult to manage compared to DU of the upper limbs. Lower limb ulcers are a rare complication of SSc, but represent a heavy clinical burden for patients. Further studies are needed to verify the classifications we have proposed and to assess the most appropriate management of SSc patients with lower limb ulcers according to their origin.

\section{REFERENCES}

1. Hachulla E, Clerson P, Launay D, Lambert M, Morell-Dubois S, Queyrel V, et al. Natural history of ischemic digital ulcers in systemic sclerosis: single-center retrospective longitudinal study. J Rheumatol 2007;34:2423-30.

2. Bruni C, Guiducci S, Bellando-Randone S, Lepri G, Braschi F, Fiori $\mathrm{G}$, et al. Digital ulcers as a "sentinel" sign for early internal organ involvement in very early systemic sclerosis. Rheumatology 2015;54:72-6

3. Guillevin L, Hunsche E, Denton CP, Krieg T, Schwierin B, Rosenberg D, et al; DUO Registry Group. Functional impairment of systemic scleroderma patients with digital ulcerations: results from the DUO Registry. Clin Exp Rheumatol 2013;31:2 Suppl 76:71-80.

4. Amanzi L, Braschi F, Fiori G, Galluccio F, Miniati I, Guiducci S, et al. Digital ulcers in scleroderma: staging, characteristics and sub-setting through observation of 1614 digital lesions. Rheumatology 2010;49:1374-82.

5. Youssef P, Brama T, Englert H, Bertouch J. Limited scleroderma is associated with increased prevalence of macrovascular disease. J Rheumatol 1995;22:469-72.

6. Hafner J, Schneider E, Burg G, Cassina PC. Management of leg ulcers in patients with rheumatoid arthritis or systemic sclerosis: the importance of concomitant arterial and venous disease. J Vasc Surg 2000;32:322-9.

7. Shanmugam VK, Price P, Attinger CE, Steen VD. Lower extremity ulcers in systemic sclerosis: features and response to therapy. Int J Rheumatol 2010;2010:747946 (E-pub August 18).

8. van den Hoogen F, Khanna D, Fransen J, Johnson SR, Baron M, Tyndall A, et al. 2013 classification criteria for systemic sclerosis: an American College of Rheumatology/European League Against Rheumatism collaborative initiative. Ann Rheum Dis 2013; 72:1747-55

9. LeRoy EC, Black C, Fleischmajer R, Jablonska S, Krieg T, Medsger TA Jr, et al. Scleroderma (systemic sclerosis): classification, subsets and pathogenesis. J Rheumatol 1988;15:202-5.

10. Allen PL, Gallagher K. The peripheral arteries. In: Allen PL, Dubbins PA, Pozniak MA, McDicken N, editors. Clinical Doppler ultrasound. 2nd ed. London: Churchill Livingstone; 2006:73-99.

11. Lau JF, Weinberg MD, Olin JW. Peripheral artery disease. Part 1: clinical evaluation and noninvasive diagnosis. Nat Rev Cardiol 2011;8:405-18.

12. Markel A, Meissner MH, Manzo RA, Bergelin RO, Strandness DE. A comparison of the cuff deflation method with Valsava's maneuver and limb compression in detecting venous valvular reflux. Arch Surg 1994;129:701-5.

13. Labropoulos N, Tiongson J, Pryor L, Tassiopoulos AK, Kang SS, Ashraf Mansour M, et al. Definition of venous reflux in the lower-extremity veins. J Vasc Surg 2003;38:793-8.

14. Dick EA, Aviv R, Francis I, Hamilton G, Baker D, Black C, et al. Catheter angiography and angioplasty in patients with scleroderma. Br J Radiol 2001;74:1091-6.

15. Cavezzi A. Duplex ultrasonography. In: Lee BB, Bergan J, Rockson S. Lymphedema: a concise compendium of theory and practice. London: Springer Science and Business Media; 2011:158-64.

16. Mekkes JR, Loots MA, Van Der Wal AC, Bos JD. Causes, investigation and treatment of leg ulceration. Br J Dermatol 2003;148:388-401.

17. Grey JE, Harding KG, Enoch S. Venous and arterial leg ulcers. BMJ 2006;11:347-50.

18. Dissemond J, Korber A, Grabbe S. Differential diagnoses in leg ulcers. J Dtsch Dermatol Ges 2006;4:627-34. Personal non-commercial use only. The Journal of Rheumatology Copyright $\subset$ 2016. All rights reserved. 
19. Silva I, Almeida J, Vasconcelos C. A PRISMA-driven systematic review for predictive risk factors of digital ulcers in systemic sclerosis patients. Autoimmun Rev 2015;142:140-52.

20. Taylor MH, McFadden JA, Bolster MB, Silver RM. Ulnar artery involvement in systemic sclerosis (scleroderma). J Rheumatol 2002;29:102-6.
21. Stucker M, Quinna S, Memmel U, Rochling A, Traupe M, Hoffman $\mathrm{K}$, et al. Macroangiopathy of the upper extremities in progressive systemic sclerosis. Eur J Med Res 2000;5:295-302.

22. Manetti M, Milia AF, Guiducci S, Romano E, Matucci-Cerinic M, Ibba-Manneschi L. Progressive loss of lymphatic vessels in skin of patients with systemic sclerosis. J Rheumatol 2011;38:297-301.

APPENDIX 1. Additional materials and methods.

Gastroesophageal reflux disease (GERD) was diagnosed when symptoms of GERD were associated to endoscopy-proven esophagitis and/or manometric abnormalities of esophageal peristalsis.

Interstitial lung disease was diagnosed by suggestive lung high-resolution computed tomography findings associated to restrictive ventilatory defect and reduced DLCO $(<70 \%)$ on pulmonary function test.

Pulmonary arterial hypertension was defined as an increase in mean pulmonary arterial pressure $\geq 25 \mathrm{mmHg}$ at rest without increase of pulmonary capillary wedge pressure (Ppcw $\leq 15 \mathrm{mmHg}$ ), assessed by right heart catheterization.

Arterial and venous color Doppler ultrasound was performed in the vascular medicine unit of our hospital by 2 experienced angiologists. Degree of arterial disease in the lower extremities was classified into 4 categories: (1) normal (0\% stenosis), (2) 1-49\% stenosis, (3) 50-99\% stenosis, and (4) total occlusion (100\% stenosis) ${ }^{11}$. Velocity criteria for the assessment of lower limb arterial stenosis were based on the peak systolic velocity (PSV) and velocity ratio (VR) when the flow velocity was normal, PSV was lower than 1.5 , and VR is $1.5: 1^{10}$. In case of a $0 \%-49 \%$ stenosis, PSV was found between 1.5 and 2, and VR is found 1.5-2:1. For a stenosis between 50-99\%, pulmonary vascular resistance was found $>2.0$ and VR was calculated as $>4: 1^{10}$. Diagnostic criteria for a hemodynamically important " $50-99 \%$ stenosis" required that the PSV was double at the lesion when compared with a more-proximal segment (it is $>200 \mathrm{~cm} / \mathrm{s}$, with evidence of turbulence) ${ }^{10}$.

Digital subtraction angiography was performed and evaluated by trained radiologists with experience $>5$ years, in the interventional radiology unit of our hospital.

Venous insufficiency was suspected on clinical examination (varicose veins, edema, hyperpigmentation, stasis dermatitis) and confirmed by venous duplex imaging combining B-mode imaging of the deep and superficial veins with pulsed Doppler flow assessment. Reflux testing was performed by cuff inflation-deflation method on the standing patient ${ }^{12}$. The cutoff value for reflux in the superficial and deep calf veins was $>500 \mathrm{~ms}$, and for the femoropopliteal veins, $>1000 \mathrm{~ms}$. Outward flow in the perforating veins were considered abnormal at greater than $350 \mathrm{~ms}^{13}$.

The modified Brewster classification was used to classify the arterial disease detected by angiography:

1. Type I: In type I disease of the lower limb there is localized aortoiliac disease with occlusive lesions involving the distal aorta as far as the common iliac arteries. In the upper limb, type I disease involves the arch of the aorta and great vessels (brachiocephalic, left common carotid, and left subclavian arteries).

2. Type II: In type II disease, initially isolated disease of the type I pattern has progressed to occlusive lesions involving the external iliac or femoral vessels.

3. Type III: Multilevel arterial disease with both proximal (above-knee) and distal (below-knee) lesions.

4. Type IV: Diseased vessels are below the knee with normal vessels above the knee.

The results were expressed as mean $\pm \mathrm{SD}$, unless otherwise indicated. For group comparisons of continuous variables, the Mann-Whitney U test was used and for categorical variables, chi-square test was used. Correlations were evaluated using Spearman's rank correlation test. Linear regression analysis was used to display a best fit line to the data. $\mathrm{P}$ value $<0.05$ was considered statistically significant. 